\title{
Propagation dynamics of vector Mathieu-Gauss beams
}

Raúl I. Hernández-Aranda, Miguel A. Bandres, Julio C. Gutiérrez Vega

Raúl I. Hernández-Aranda, Miguel A. Bandres, Julio C. Gutiérrez Vega, "Propagation dynamics of vector Mathieu-Gauss beams," Proc. SPIE 6290, Laser Beam Shaping VII, 629011 (9 September 2006); doi:

10.1117/12.679737

SPIE. Event: SPIE Optics + Photonics, 2006, San Diego, California, United States 


\title{
Propagation dynamics of vector Mathieu-Gauss beams
}

\author{
Raúl I. Hernández-Aranda ${ }^{a}$, Miguel A. Bandres ${ }^{b}$, and Julio C. Gutiérrez Vega ${ }^{a}$ \\ ${ }^{a}$ Photonics and Mathematical Optics Group, Tecnológico de Monterrey, Monterrey, México \\ 64849 \\ ${ }^{b}$ California Institute of Technology, Pasadena CA 91125, USA
}

\begin{abstract}
The vector Mathieu-Gauss beams of integer order are examined as the solutions of the vector paraxial wave equation in elliptical coordinates. The propagation of the vector components and the three-dimensional intensity distribution of focused vector Mathieu-Gauss beams are analyzed for a variety of polarizations. Conditions in which the linearly polarized Mathieu-Gauss beams can be approximated by the scalar solutions of the paraxial wave equation are also discussed.
\end{abstract}

Keywords: Vector paraxial wave equation, Mathieu-Gauss beams, Polarization.

\section{INTRODUCTION}

Vector Helmholtz-Gauss beams (vHzG) have been recently introduced by Bandres and Gutiérrez-Vega. ${ }^{1}$ These beams constitute localized solutions of the vector paraxial wave equation (vPWE). These solutions represent a monochromatic field propagating along the positive $z$ axis, and have a transverse distribution whose amplitude is modulated by a Gaussian factor. Vector solutions of the paraxial wave equation have been studied for some time by different authors. ${ }^{2-4}$

In this article we study the propagation dynamics of vector Mathieu-Gauss beams and their polarization properties. Two general cases are considered in this study, namely unperturbed free-space propagation, and the propagation after a thin converging lens. We have paid special attention to the issue of polarization.

\section{VECTOR HELMHOLTZ-GAUSS BEAMS}

Consider the propagation of a monochromatic electromagnetic field along the positive $z$ axis. The expression for the electric and magnetic fields can be written as $\mathbf{E}=\left(\mathbf{E}_{t}+\hat{\mathbf{z}} E_{z}\right) \exp (i k z)$ and $\mathbf{H}=\left(\mathbf{H}_{t}+\hat{\mathbf{z}} H_{z}\right) \exp (i k z)$ respectively, where $k=\omega\left(\mu_{0} \epsilon_{0}\right)^{1 / 2}=\sqrt{k_{t}^{2}+k_{z}^{2}}$ is the wave number, and the subscripts $t$ and $z$ refer to transverse and longitudinal components.

It is well known that in free space, Maxwell's equations demand the transversality between the electric and magnetic fields; therefore, once the electric or the magnetic field is computed, the remaining field can readily be obtained by using $\mathbf{H}_{t}=\left(\epsilon_{0} / \mu_{0}^{1 / 2}\right) \hat{\mathbf{z}} \times \mathbf{E}_{t}$.

Following the analysis of Ref.[1], the transverse component of the electric field $\mathbf{E}$, namely $\mathbf{E}_{t}$ is assumed to be of the form

$$
\mathbf{E}_{t}=\mathbf{U}(X, Y, \zeta) G(\mathbf{r})
$$

where $\mathbf{r}$ is the usual radial vector in polar coordinates, $(X, Y)=(x / \zeta, y / \zeta)$ are scaled cartesian coordinates, and $\zeta(z)=1+i z / z_{R}$. The factor $G(\mathbf{r})$ represents a Gaussian amplitude modulation given by $G(\mathbf{r})=\zeta^{-1} \exp \left(-r^{2} / w_{0}^{2} \zeta\right)$, and function $\mathbf{U}$ is in turn expressed as the product between a transverse amplitude function $\boldsymbol{\Psi}(X, Y)$, and a function $Z(\zeta)=\exp \left[k_{t}^{2} w_{0}^{2}\left(\zeta^{-1}-1\right) / 4\right]$ depending only on the propagation coordinate. The function $\boldsymbol{\Psi}$ is known to be a solution to the vector Helmholtz equation in $2 \mathrm{D}$, and it can represent two independent vector solutions of the form: $:^{1,5}$

Further author information: (Send correspondence to J.C.G.V. or R.I.H.A.)

J.C.G.V.: E-mail: juliocesar@itesm.mx,

R.I.H.A.: E-mail: raul.aranda@itesm.mx

Laser Beam Shaping VII, edited by Fred M. Dickey, David L. Shealy, Proceedings

of SPIE Vol. 6290, 629011, (2006) · 0277-786X/06/\$15 - doi: 10.1117/12.679737

Proc. of SPIE Vol. $6290629011-1$ 


$$
\Psi^{(1)}=\nabla_{T} W(X, Y), \quad \Psi^{(2)}=-\hat{z} \times \Psi^{(1)}
$$

where the function $W(X, Y)$ is a solution of the 2D scalar Helmholtz equation. In general, it is seen that the transverse distribution of ${ }_{\mathrm{vHzG}}$ beams is completely determined by the nature of the $W$ function. The expressions for the two classes of vector beam solutions of the vPWE in terms of $W$ are

$$
\begin{aligned}
\mathbf{E}_{t}^{(1)} & =Z(\zeta) G(\mathbf{r}) \nabla_{T} W, \\
E_{z}^{(1)} & =-\frac{i Z(\zeta) G(\mathbf{r})}{\zeta}\left(\frac{k_{t}^{2}}{k} W+\frac{2}{k \omega_{0}} \nabla_{T} W \cdot \frac{\mathbf{r}_{t}}{\omega_{0}}\right), \\
\mathbf{H}_{t}^{(1)} & =\sqrt{\frac{\epsilon_{0}}{\mu_{0}}} Z(\zeta) G(\mathbf{r})\left(\hat{z} \times \nabla_{T} W\right), \\
H_{z}^{(1)} & =-\sqrt{\frac{\epsilon_{0}}{\mu_{0}}} \frac{2 i}{k \omega_{0}} \frac{Z(\zeta) G(\mathbf{r})}{\zeta}\left(\hat{z} \times \nabla_{T} W\right) \cdot \frac{\mathbf{r}_{t}}{\omega_{0}},
\end{aligned}
$$

for the first-class, corresponding to the TM solution, whereas for the second class or TE solution, the expressions read

$$
\begin{aligned}
\mathbf{E}_{t}^{(2)} & =-Z(\zeta) G(\mathbf{r})\left(\hat{z} \times \nabla_{T} W\right), \\
E_{z}^{(2)} & =\frac{2 i}{k \omega_{0}} \frac{Z(\zeta) G(\mathbf{r})}{\zeta}\left(\hat{z} \times \nabla_{T} W\right) \cdot \frac{\mathbf{r}_{t}}{\omega_{0}} \\
\mathbf{H}_{t}^{(2)} & =\sqrt{\frac{\epsilon_{0}}{\mu_{0}}} Z(\zeta) G(\mathbf{r}) \nabla_{T} W \\
H_{z}^{(2)} & =-\sqrt{\frac{\epsilon_{0}}{\mu_{0}}} \frac{i Z(\zeta) G(\mathbf{r})}{\zeta}\left(\frac{k_{t}^{2}}{k} W+\frac{2}{k \omega_{0}} \nabla_{T} W \cdot \frac{\mathbf{r}_{t}}{\omega_{0}}\right) .
\end{aligned}
$$

The scalar function $W(X, Y)$ may also be expressed in integral form using the Whittaker representation

$$
W(X, Y)=\int_{-\pi}^{\pi} A(\varphi) \exp \left[i k_{t}(X \cos \varphi+Y \sin \varphi)\right] d \varphi,
$$

where the function $A(\varphi)$ represents the angular spectrum of $W$, and the transverse and longitudinal components of the wave vector satisfy the relation $k^{2}=k_{t}^{2}+k_{z}^{2}$.

\section{VECTOR MATHIEU-GAUSS BEAMS}

One of the most relevant results from Ref.[1], is that expressions derived for vHzG beams are of a general character, this means they are independent of the coordinate system one chooses to work with. It can be readily seen from Eqs.(3) and (7) how the transverse structure and vectorial nature of the field are determined by $\nabla_{T} W$.

From the infinite number of possible solutions for $W$, of particular interest are the ones constituting families of orthogonal eigenfunctions of the two dimensional Helmholtz equation. In circular coordinates the eigenfunctions are $J_{m}\left(k_{t} r\right) \exp (i m \varphi)$ and lead to the case of vector Bessel-Gauss beams, which have been previously studied by Hall. ${ }^{4}$

In the elliptic cylindrical coordinate system the eigenfunctions $W$ correspond to Mathieu functions, ${ }^{6,7}$ which in turn give rise to vector Mathieu-Gauss (vMG) beams. Given in terms of the $m$-th order radial and angular (even and odd) Mathieu functions $W$ read as 


$$
\begin{aligned}
& W_{m}^{e}(\xi, \eta)=\mathrm{Je}_{m}(\xi, q) \mathrm{ce}_{m}(\eta, q) \\
& W_{m}^{o}(\xi, \eta)=\mathrm{Jo}_{m}(\xi, q) \mathrm{se}_{m}(\eta, q)
\end{aligned}
$$

where the superscripts $e, o$ stand for even and odd solutions respectively, whereas the functions $\mathrm{Je}_{m}(\cdot), \mathrm{Jo}_{m}(\cdot)$ represent even and odd radial Mathieu functions, accordingly the even and odd angular Mathieu functions are given by $\operatorname{ce}_{m}(\cdot), \operatorname{se}_{m}(\cdot)$, subscript $m$ is a non-negative integer number indicating the order of the functions and $q$ is a free parameter commonly referred to as the ellipticity parameter.

As we have mentioned earlier, the transverse structure of vMG beams is primarily determined by the gradient of the function $W$, which in the scaled elliptic coordinates is

$$
\nabla_{T} W(\bar{\xi}, \bar{\eta})=\left(\frac{1}{h_{\bar{\xi}}} \frac{\partial}{\partial \bar{\xi}} \hat{\xi}+\frac{1}{h_{\bar{\eta}}} \frac{\partial}{\partial \bar{\eta}} \hat{\eta}\right) W(\bar{\xi}, \bar{\eta})
$$

where $h_{\bar{\xi}}=h_{\bar{\eta}}=f_{o} \sqrt{\sinh ^{2} \bar{\xi}+\sin ^{2} \bar{\eta}}, f_{o}$ is the semifocal separation at the waist plane $z=0$, and the connection between cartesian and elliptic variables is given by the relations $(X, Y)=\left(f_{0} \cosh \bar{\xi} \cos \bar{\eta}, f_{0} \sinh \bar{\xi} \sin \bar{\eta}\right)$.

For numerical purposes it is convenient to employ the cartesian gradient of $W(\bar{\xi}, \bar{\eta})$, since it directly provides expressions for the $\hat{\mathbf{x}}$ and $\hat{\mathbf{y}}$ components. This gradient is calculated as follows

$$
\begin{aligned}
\nabla_{T} W(\bar{\xi}, \bar{\eta}) & =\frac{\partial}{\partial X} W(\bar{\xi}, \bar{\eta}) \hat{\mathbf{x}}+\frac{\partial}{\partial Y} W(\bar{\xi}, \bar{\eta}) \hat{\mathbf{y}} \\
\frac{\partial}{\partial X} W(\bar{\xi}, \bar{\eta}) & =\frac{f_{o} \sinh \bar{\xi} \cos \bar{\eta}}{h_{\bar{\xi}}^{2}} \frac{\partial}{\partial \bar{\xi}} W(\bar{\xi}, \bar{\eta})-\frac{f_{o} \cosh \bar{\xi} \sin \bar{\eta}}{h_{\bar{\eta}}^{2}} \frac{\partial}{\partial \bar{\eta}} W(\bar{\xi}, \bar{\eta}) \\
\frac{\partial}{\partial Y} W(\bar{\xi}, \bar{\eta}) & =\frac{f_{o} \cosh \bar{\xi} \sin \bar{\eta}}{h_{\bar{\xi}}^{2}} \frac{\partial}{\partial \bar{\xi}} W(\bar{\xi}, \bar{\eta})+\frac{f_{o} \sinh \bar{\xi} \cos \bar{\eta}}{h_{\bar{\eta}}^{2}} \frac{\partial}{\partial \bar{\eta}} W(\bar{\xi}, \bar{\eta})
\end{aligned}
$$

for the sake of space we will restrict ourselves to the use of an even Mathieu function of the form given in Eq.(12), for all numerical calculations and analysis. Additionally, the derivation of all results will be performed taking a TM polarized vMG beam, without loss of generality, since the corresponding results for TE solutions can be straightforwardly calculated from the orthogonality relations between TM and TE modes stated in Eq.(2).

\section{POLARIZATION PROPERTIES OF VECTOR MATHIEU GAUSS BEAMS}

The general form of a vMG beam consists of a superposition between TM and TE solutions, i.e. $\mathbf{E}=\alpha \mathbf{E}^{(1)}+$ $\beta \mathbf{E}^{(2)}$, where $(\alpha, \beta)$ are arbitrary and in general complex constants. On the other hand, it is possible to express the gradient operator in Eq.(14) in several orthogonal coordinate systems, and consequently different polarization basis may be used to decompose the field $\mathbf{E}$ at any point in space into two orthogonal transverse parts. For instance if we choose the circular polarization basis vectors $\hat{\mathbf{u}}^{ \pm}=(\hat{\mathbf{x}} \pm i \hat{\mathbf{y}}) / \sqrt{2}$ then we may express the gradient of Eq.(15) in this basis, and the following can easily be verified

$$
\begin{aligned}
\nabla_{T} W & =\frac{1}{\sqrt{2}}\left(\frac{\partial W}{\partial X}-i \frac{\partial W}{\partial Y}\right) \hat{\mathbf{u}}^{+}+\frac{1}{\sqrt{2}}\left(\frac{\partial W}{\partial X}+i \frac{\partial W}{\partial Y}\right) \hat{\mathbf{u}}^{-} \\
-\hat{\mathbf{z}} \times \nabla_{T} W & =\frac{i}{\sqrt{2}}\left(\frac{\partial W}{\partial X}-i \frac{\partial W}{\partial Y}\right) \hat{\mathbf{u}}^{+}-\frac{i}{\sqrt{2}}\left(\frac{\partial W}{\partial X}+i \frac{\partial W}{\partial Y}\right) \hat{\mathbf{u}}^{-}
\end{aligned}
$$


therefore a right or left-circularly polarized beam can be calculated directly by setting $(\alpha, \beta)=(1, \pm i)$ respectively, which in turn will lead to

$$
\begin{aligned}
\mathbf{E}^{ \pm} & =\mathbf{E}^{(1)} \pm i \mathbf{E}^{(2)} \\
& =\sqrt{2} Z(\zeta) G(\mathbf{r})\left(\frac{\partial W}{\partial X} \mp i \frac{\partial W}{\partial Y}\right) \hat{\mathbf{u}}^{ \pm}
\end{aligned}
$$

the differential operator in the previous equation can be used to construct a new scalar field also satisfying the 2D scalar Helmholtz equation, namely $\partial^{ \pm} W=(\partial / \partial X \pm i \partial / \partial Y) W$, making use of the latter and employing the circular basis vectors one can build up linearly polarized fields, for instance a linearly polarized vMG beam in the $\hat{\mathbf{x}}$ direction is obtained with the following superposition

$$
\begin{aligned}
\mathbf{E} & =\frac{\sqrt{(2)}}{2} Z(\zeta) G(\mathbf{r})\left[\partial^{+} W \hat{\mathbf{u}}^{+}+\partial^{+} W \hat{\mathbf{u}}^{-}\right] \\
& =Z(\zeta) G(\mathbf{r}) \partial^{+} W \hat{\mathbf{x}}
\end{aligned}
$$

linearly polarized fields in other directions are possible by a suitable choice of the basis vectors and superposition of vMG beams.

\section{PROPAGATION OF VECTOR MATHIEU-GAUSS BEAMS}

Propagation of a vMG beam in free space is described by Eqs. (3) through (10). In our subsequent analysis we only consider the transverse component of the vMG beam, since the longitudinal part has no effect on the transverse structure or polarization profile.

A general picture of the propagation properties of vMG beams can be acquired if one consider that the vMG beam is formed as a coherent superposition of vector Gaussian beams ${ }^{7,8}$ having their waist planes coincident at $z=0$, their mean propagation axis lying on the surface of a cone with a half-aperture angle $\theta_{0} \approx \arcsin \left(k_{t} / k\right) \approx$ $k_{t} / k$ and whose amplitude is modulated by the angular function $A(\varphi)$. Therefore the propagation characteristics of the constituent vector beams is governed by the conical spreading of the wavefront and diffraction of the constituent vector Gaussian beams.

Two important parameters characterize the propagation of a vMG beam, namely the transverse component of the wave vector $k_{t}$ and the waist of the Gaussian beam $w_{0}$. A simple inspection permit us to verify that when $w_{0} \rightarrow \infty$, vMG beams reduce to pure nondiffracting vector Mathieu beams, on the other hand when the function $W$ becomes a constant, i.e. $k_{t}$ tends to zero, vMG beams reduce to pure vector Gaussian beams.

By introducing the parameter $\gamma=k_{t} w_{0} / 2$ one can identify three important cases, when $\gamma \ll 1$ because $k_{t} \rightarrow 0$ the dominant propagation characteristics are imposed by the Gaussian beams; for the case when $\gamma \gg 1$ the nondiffracting behavior introduced by the function $W$ prevails, and a significant superposition of the constituent vector Gaussian beams survives up to a distance $z_{\max } \approx w_{0} k / k_{t}=z_{R} / \gamma$, whereas the case $\gamma \approx 1$ corresponds to the transition between both cases.

Transverse profiles of the TE and TM electric fields of a vMG beam are shown for even Mathieu functions $W_{m}^{e}$ of different orders, and different values of $q$. The parameter $q$ carries information about the ellipticity of the coordinate system and of the transverse spatial frequency $k_{t}$, explicitly it is calculated as $q=f_{0}^{2} k_{t}^{2} / 4$, where $f_{0}$ is the interfocal separation of the elliptical coordinate system at $z=0$. When $q$ tends to zero, the elliptic system approaches to circular coordinates, therefore the transverse patterns possess circular symmetry. Results of the simulations for this special case are shown in Fig. 1 and are consistent with those reported by Greene and Hall ${ }^{9}$ for vector Bessel-Gauss beams, the beam possesses an on-axis null and is azimuthally polarized.

As one increases the ellipticity parameter, vMG beams elliptical nature become more evident since circular symmetry is broken, and nodal lines along the $\eta$ coordinate appear. We have illustrated this fact in Fig. 2, note that azimuthal polarization is lost, now the fields contain both azimuthal and radial components. 


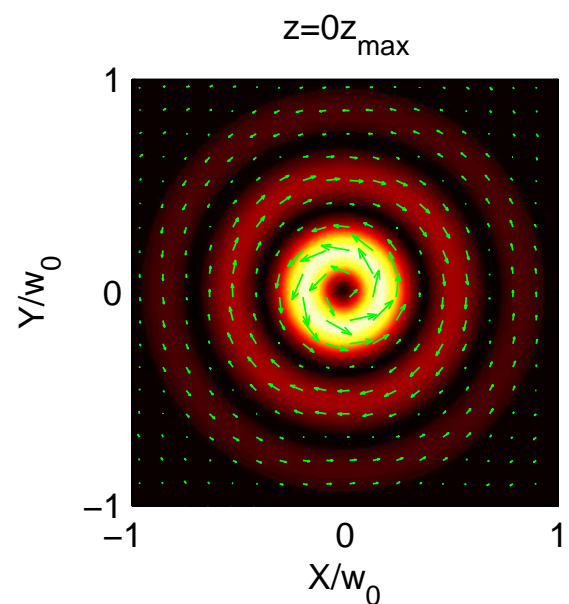

(a)

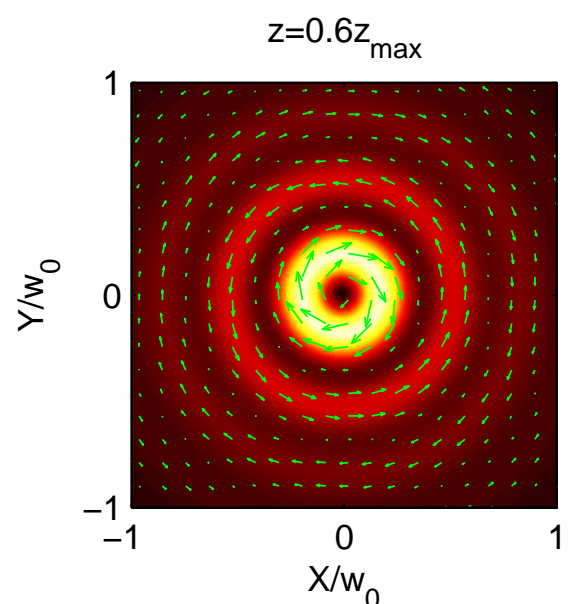

(b)

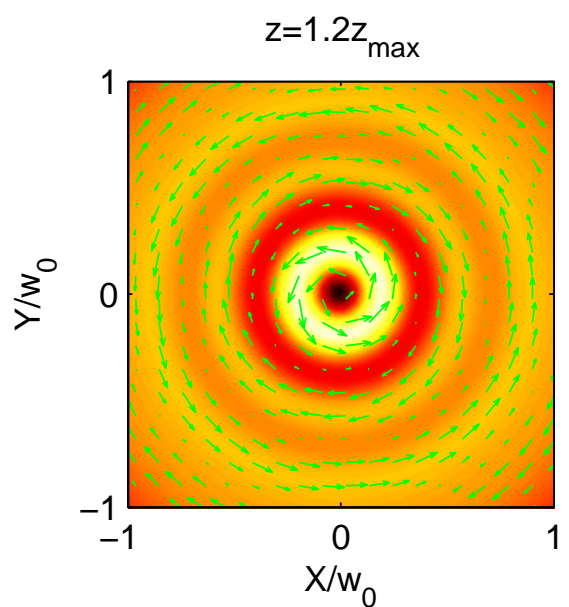

(c)

Figure 1. 2D Intensity profile $|E|^{2}$ of a TE polarized vector Mathieu-Gauss beam at different propagation distances. Profiles correspond to a function $W_{0}^{e}(\bar{\xi}, \bar{\eta}, q=0)$, and $\gamma=5$.

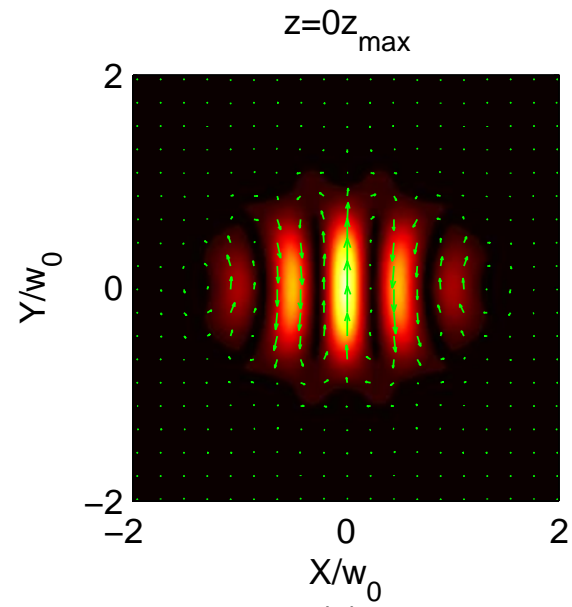

(a)

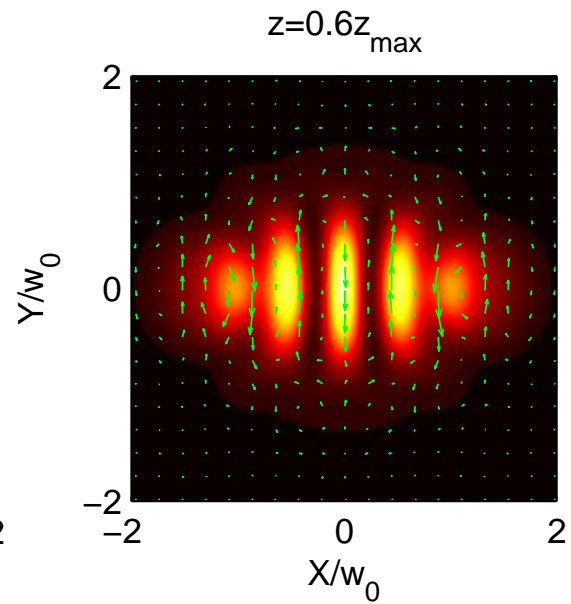

(b)

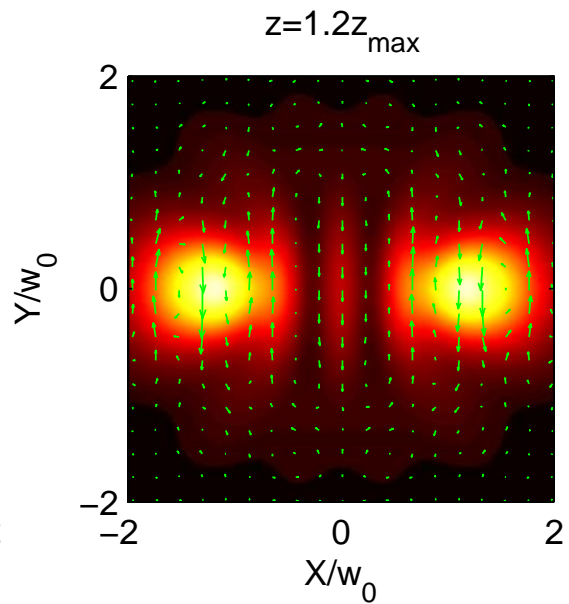

(c)

Figure 2. 2D Intensity profile $|E|^{2}$ of a TE polarized vector Mathieu-Gauss beam at different propagation distances. Profiles correspond to a function $W_{5}^{e}(\bar{\xi}, \bar{\eta}, q=15)$, and $\gamma=3$.

\section{ANGULAR SPECTRUM OF VECTOR MATHIEU-GAUSS BEAMS}

The angular spectrum of a $\mathrm{vHzG}$ beam, Eq.(3), across an arbitrary plane at a distance $z$ from the origin and parallel to the $(x, y)$ plane can be computed by means of the two-dimensional Fourier transform

$$
\mathcal{U}(u, v ; \zeta)=\frac{1}{2 \pi} \int_{-\infty}^{\infty} \int_{-\infty}^{\infty} \zeta \mathbf{E}_{t}^{T M}(x, y ; \zeta) \exp [-i(x u+y v)] \mathrm{dxdy}
$$

where $(u, v)$ are the cartesian coordinates in the frequency space. After performing this integral one obtains the expression for the angular spectrum of an arbitrary $\mathrm{vHzG}$ beam, namely 


$$
\mathcal{U}(u, v ; \zeta)=D(\zeta) \exp \left(\frac{\zeta \tilde{\rho}^{2}}{w_{0}^{2}}\right) \tilde{\nabla}_{t} W(\tilde{u}, \tilde{v})
$$

where $\tilde{\rho}^{2}=\tilde{u}^{2}+\tilde{v}^{2}$, and $(\tilde{u}, \tilde{v})=\left(w_{0}^{2} u / 2 i, w_{0}^{2} v / 2 i\right)$. The tilde over the gradient operator indicates that differentiation is with respect to the tilded variables. Finally, our $D(\zeta)$ function is defined as

$$
D(\zeta)=\frac{w_{0}^{2}}{2} Z(\zeta) \exp \left(\frac{-w_{0}^{2} k_{t}^{2}}{4 \zeta}\right)
$$

and is a complex amplitude factor depending only on $z$ through the complex parameter $\zeta$. For vMG beams we have $W(\tilde{u}, \tilde{v})=W_{m}^{e}(\tilde{\xi}, \tilde{\eta} ; q)=\operatorname{Je}_{m}(\tilde{\xi}, q) \mathrm{ce}_{m}(\tilde{\eta}, q)$, and the complex elliptic variables are determined by

$$
\begin{aligned}
& \tilde{u}=f_{0} \cosh \tilde{\xi} \cos \tilde{\eta} \\
& \tilde{v}=f_{0} \sinh \tilde{\xi} \sin \tilde{\eta}
\end{aligned}
$$

Expressions for TE solutions are directly derived from the duality property of the electromagnetic fields.

\section{FOCUSING OF VECTOR MATHIEU-GAUSS BEAMS}

We now turn our attention to the case of having a vMG beam at the input plane of a non-apertured focusing thin lens. Assuming a TM vector Mathieu-Gauss beam as the input field, and employing the Huygens-Fresnel diffraction integral, one can obtain the propagation of the focused vMG beam along the longitudinal coordinate, which is given by

$$
\mathbf{E}^{T M}(\tilde{\xi}, \tilde{\eta}, z)=\frac{k}{i 2 a^{2} z} \exp \left[i k\left(z+\frac{r^{2}}{2 z}\right)\right] \exp \left[-\frac{1}{4 a^{2}}\left(k_{t}^{2}+\frac{k^{2} r^{2}}{z^{2}}\right)\right] \tilde{\nabla}_{t} W(\tilde{\xi}, \tilde{\eta})
$$

where $r^{2}=x^{2}+y^{2}$ is the radial coordinate, but now the complex elliptic variables $(\tilde{\xi}, \tilde{\eta})$ are determined by the following relations

$$
\begin{aligned}
& x=\frac{2 i a^{2} z f_{0}}{k} \cosh \tilde{\xi} \cos \tilde{\eta} \\
& y=\frac{2 i a^{2} z f_{0}}{k} \sinh \tilde{\xi} \cos \tilde{\eta}
\end{aligned}
$$

the transverse gradient operator $\tilde{\nabla}_{t}$ is again defined over the complex elliptic variables according to Eq.(14), and the parameter $a^{2}$ is

$$
a^{2}=\left[\frac{1}{w_{0}^{2}}-\frac{i k}{2}\left(\frac{1}{z}-\frac{1}{f}\right)\right]
$$

where $w_{0}$ is the Gaussian beam waist at $z=0, k$ the wave number, $k_{t}$ the magnitude of the transverse component of the wave vector, and $f$ is the lens focal distance. The function $W(\tilde{\xi}, \tilde{\eta})$ can be either of Eqs.(12) or (13). For the sake of space we will only consider $W_{m}^{e}(\tilde{\xi}, \tilde{\eta})=\operatorname{Je}_{m}(\tilde{\xi}, q) \mathrm{ce}_{m}(\tilde{\eta}, q)$ in our simulations, which corresponds to an even Mathieu function of $m$-th order. As expected, the introduction of the thin lens affects the propagation of the vMG beams only by scaling the radial coordinate and propagation distance. It can be easily verified that, with exception of a quadratic phase factor, Eq.(22) reduces to Eq.(20) when $z=f$.

It is known that when a HzG beam is focused by a lens, an annular distribution is obtained at the focal plane ${ }^{10}$ this striking result is a consequence of the wave nature of $\mathrm{HzG}$ beams. Our simulations of focused 


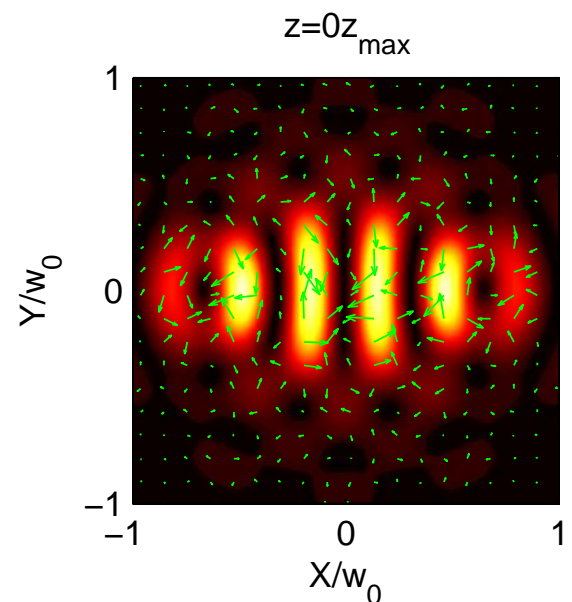

(a)

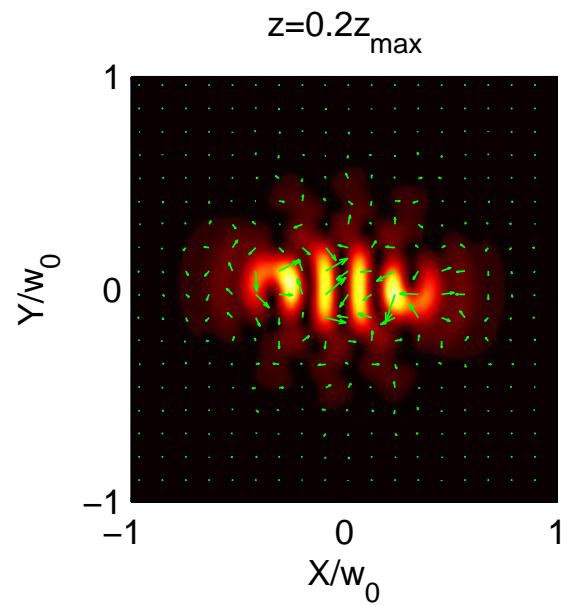

(b)

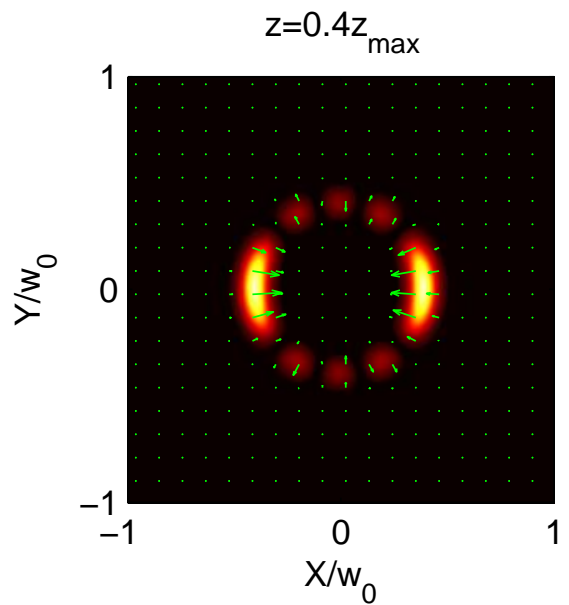

(c)

Figure 3. 2D Intensity profile $|E|^{2}$ of a left-circularly polarized vMG beam at different propagation distances. Profiles correspond to a function $W_{4}^{e}(\bar{\xi}, \bar{\eta}, q=10)$, and $\gamma=5$.
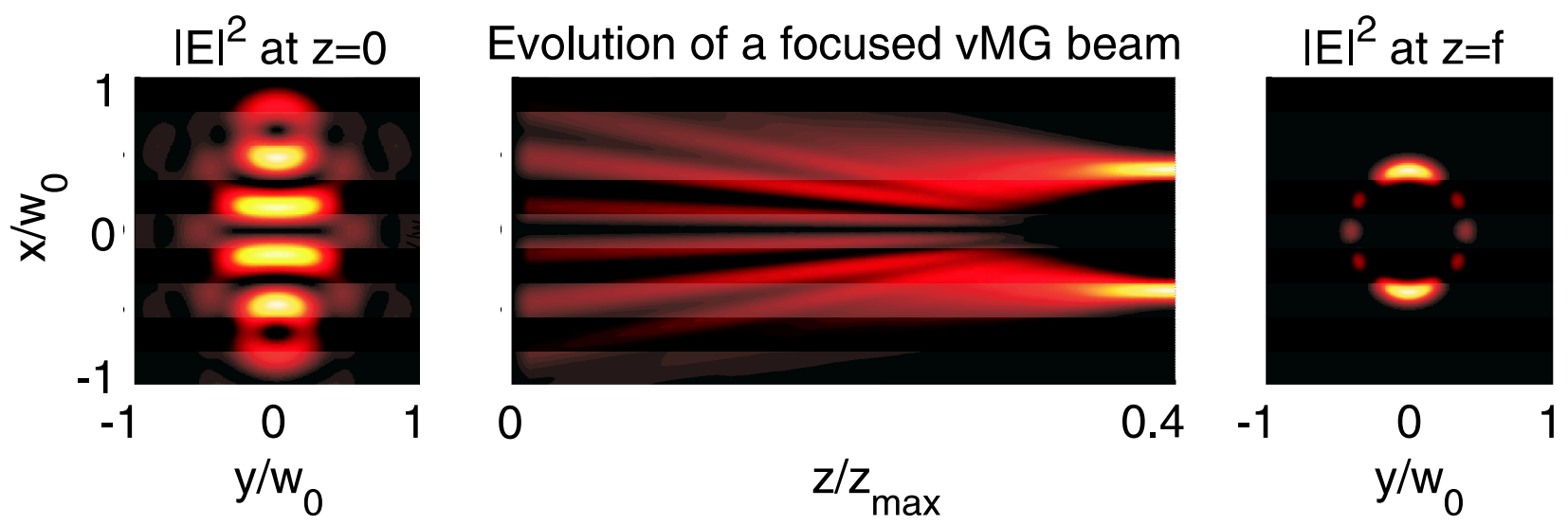

Figure 4. Evolution of the focused vMG from Fig. 3(a). Propagation goes from plane $z=0$ where the lens is located, to the focal plane $z=f=0.4 z_{\max }$. Observe the formation of intensity peaks at the focal plane. This confirms the nondiffracting behavior of vMG beams for $\gamma \gg 1$. 


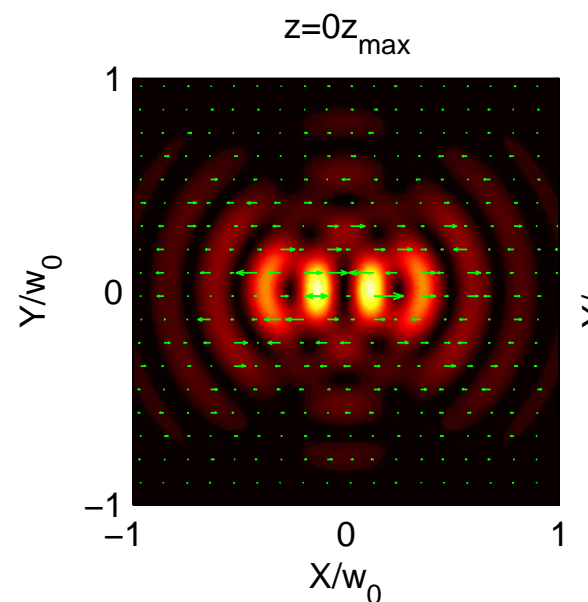

(a)

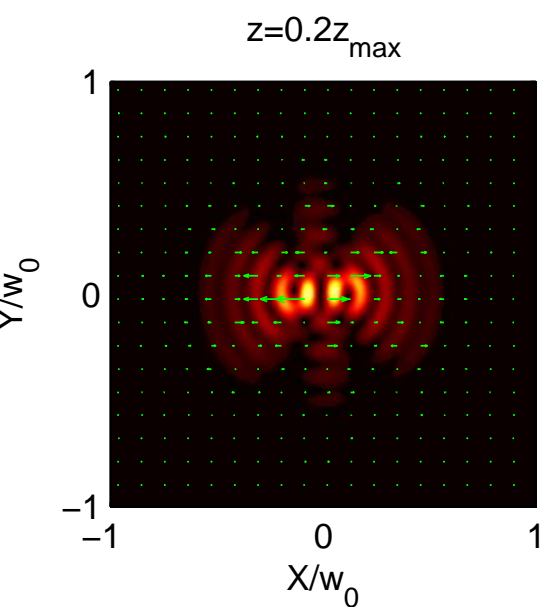

(b)

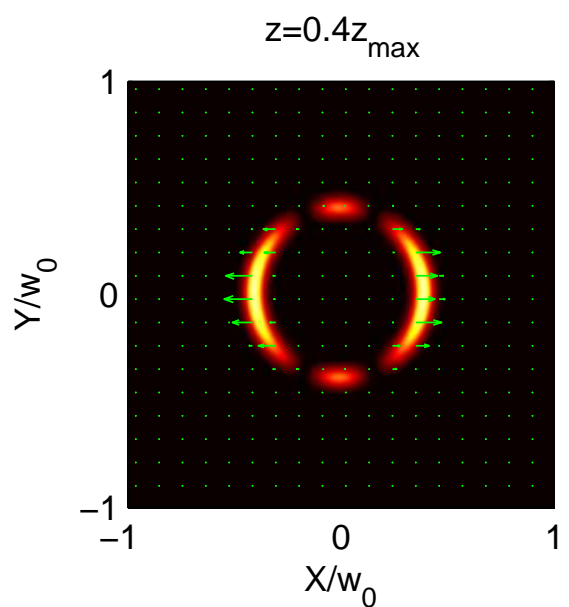

(c)

Figure 5. 2D Intensity profile $|E|^{2}$ of a linearly polarized vMG beam at different propagation distances. Simulation parameters and function are: $W_{2}^{e}(\bar{\xi}, \bar{\eta}, q=4)$, and $\gamma=7$. Note that the field preserves its polarization state along propagation.

vector Mathieu-Gauss beams support the latter, the following simulation parameters are chosen $w_{0}=3 \mathrm{~mm}$, $\lambda=632.8 \mathrm{~nm}$, and $\gamma=5$, Fig. 3 shows the intensity profiles of a focusing left-circularly polarized vMG beam at different $z$ planes for a $W_{4}^{e}$ Mathieu function. The lens is located at $z=0$ and the focal distance is $f=0.4 z_{\max }$. It is clearly evident how the field evolves to form an annular distribution at the focal plane, this is also an expected behavior since as we have mentioned earlier when $\gamma \gg 1$ the nondiffracting properties of the vMG beam become dominant.

In order to show the evolution of the focused vMG beam in Fig. 3 we have simulated the propagation of the initial field, Fig. 3(a), from plane $z=0$ to $z=f$, and divided the propagation interval into 100 planes equally spaced. Fig. 4 shows the formation of the intensity peaks at the focal plane, confirming again the nondiffracting properties of vMG beams.

Finally, Fig. 5 illustrates the case of a linearly polarized field according to Eq.(18), where it is evident that the field preserves its polarization state as expected. The function employed in this simulation is $W_{2}^{e}(\tilde{\xi}, \tilde{\eta}, q=4)$, with $w_{0}=3 \mathrm{~mm}$ and $\gamma=7$.

\section{CONCLUSIONS}

In this paper, we have studied the propagation properties of vector Mathieu-Gauss beams. Starting from the general expressions for vector Helmholtz-Gauss beams ${ }^{1}$ we deduced closed-form expressions for the angular spectrum and propagation of focused $\mathrm{vHzG}$ beams in general, and for $\mathrm{vMG}$ in particular. Additionally, conditions in which the linearly polarized Mathieu-Gauss beams can be approximated by the scalar solutions of the paraxial wave equation were discussed. The linearly polarized vMG beam has been constructed employing the basis vectors of circular polarization, other types of polarizations can be obtained by a suitable superposition of vMG beams. Finally, it was also shown that the angular spectrum of vMG corresponds to a modulated ring in the frequency space, which accounts for the nondiffracting nature of these beams.

\section{ACKNOWLEDGMENTS}

This research was supported by Consejo Nacional de Ciencia y Tecnología (grant 42808) and by the Tecnológico de Monterrey (grant CAT-007). 


\section{REFERENCES}

1. M. A. Bandres and J. C. Gutiérrez-Vega, "Vector Helmholtz-Gauss and vector Laplace-Gauss beams," Opt. Lett. 30, 2155-2157 (2005).

2. M. Lax, W. H. Louisell, and W. B. McKnight, "From Maxwell to paraxial wave optics," Phys. Rev. A 11, 1365-1370 (1975).

3. L. W. Davis and G. Patsakos, "TE and TM electromagnetic beams in free space," Opt. Lett. 6, 22-23 (1981).

4. D. G. Hall, "Vector-beam solutions of Maxwell's wave equation ," Opt. Lett. 21, 9-11 (1996).

5. J. A. Stratton, Electromagnetic Theory, (McGraw-Hill, New York, 1941).

6. J. C. Gutiérrez-Vega, M. D. Iturbe-Castillo, and S. Chávez-Cerda, "Alternative formulation for invariant optical fields," Opt. Lett. 25, 1493 (2000).

7. J. C. Gutiérrez-Vega and M. A. Bandres, "Helmholtz-Gauss waves," J. Opt. Soc. Am. A 22, 289-298 (2005).

8. F. Gori, G. Guattari and C. Padovani, "Bessel-Gauss beams," Opt. Commun. 64, 491-495 (1987).

9. P. L. Greene and D. G. Hall, "Properties and diffraction of vector Bessel-Gauss beams," J. Opt. Soc. Am. A 15, 3020-3027 (1998).

10. J. C. Gutiérrez-Vega, R. Rodríguez-Masegosa and S. Chávez-Cerda, "Focusing evolution of generalized propagation invariant optical fields," J. Opt. A: Pure Appl. Opt. 5, 276-282 (2003). 